\title{
Immunotherapy for Merkel cell carcinoma: a turning point in patient care
}

\author{
Isaac S. Chan', Shailender Bhatia ${ }^{2}$, Howard L. Kaufman ${ }^{3}$ and Evan J. Lipson ${ }^{1,4^{*}}$
}

\begin{abstract}
Merkel Cell carcinoma (MCC) is a rare but aggressive cancer, with an estimated disease-associated mortality as high as $46 \%$. MCC has proven to be an immunologically responsive disease and the advent of immune checkpoint inhibitors has changed the treatment landscape for patients with advanced MCC. In this review, we discuss the rationale for the use of immune checkpoint inhibition, review current single agent therapies tested in and approved for MCC, and discuss emerging immunotherapeutic options for these patients.
\end{abstract}

\section{Background}

Merkel cell carcinoma (MCC) is a neuroendocrine-like tumor of the skin. First described in 1972, it is a rare diagnosis, with an annual incidence of approximately 0.6 out of 100,000 persons in the United States [1]. A high index of suspicion is required for diagnosis given its rarity and often inconspicuous presentation as a small, asymptomatic ulcerated, cystic or acneiform lesion [2]. The incidence of the disease has quadrupled since 1986, perhaps due the development of more sophisticated diagnostic tools, an aging population, and increasing use of therapeutic immunosuppression [3, 4]. Age, immunosuppression, and sun exposure remain the largest risk factors for this deadly disease, with an estimated disease-associated mortality of $33 \%$ to $46 \%$ [5]. In patients with localized MCC, the five-year overall survival rate is $55.6 \%$. In patients with advanced disease, historical five-year survival are $35.4 \%$ for those with regional nodal disease and 13.5\% for those with distant metastases [6]. These survival rates reveal the historical lack of effective treatment options for patients with MCC [7]. However, recent advances in our understanding of the biology of MCC have created opportunities for novel therapeutic strategies and hope for improving treatment efficacy. For example, the discovery of the oncogenic Merkel cell polyomavirus (MCPyV) that is

\footnotetext{
* Correspondence: evanlipson@jhmi.edu

'Department of Oncology, Johns Hopkins University School of Medicine, Sidney Kimmel Comprehensive Cancer Center, and Bloomberg-Kimmel Institute for Cancer Immunotherapy, Baltimore, MD, USA

${ }^{4}$ Melanoma and Cancer Immunology Programs, Johns Hopkins University School of Medicine, 1550 Orleans Street, Room 507, Baltimore, MD 21287, USA

Full list of author information is available at the end of the article
}

associated with approximately $80 \%$ of $\mathrm{MCC}$ cases has led to further investigations into whether dysregulated immune surveillance plays a role in MCC pathogenesis, and how best to generate anti-tumor immunity [8]. Recent results from clinical trials of immune checkpoint inhibitors suggest that these therapies could improve treatment outcomes by unleashing anti-tumor immunity against an immunogenic tumor. In this review, we discuss the spectrum of therapeutic options for MCC and the pivotal role that immune checkpoint inhibition might play in improving patient outcomes.

Patients with primary, or localized MCC, which accounts for $65-70 \%$ of patients at diagnosis, typically undergo surgical resection followed by adjuvant radiotherapy to prevent recurrence at the primary site and involved regional lymphatics [6]. Even after definitive therapy of stage I and II disease, the potential for recurrence is high, with recurrence rates of $35 \%$ at three years [9]. In a case series of 237 MCC patients with local or regional disease, median time-to-recurrence was 9 months (range, 270 months) and $91 \%$ of the recurrences occurred within 2 years of initial diagnosis [10]. Study data do not support the routine use of adjuvant systemic chemotherapy for high-risk resected MCC and, therefore, adjuvant chemotherapy is not included in the NCCN guidelines. Without evidence demonstrating a clear survival benefit, the risks of immunosuppression, toxicity and diminished quality of life are not justified [11].

Until recently, chemotherapy has been a mainstay of therapy for patients with advanced MCC. Because MCC bears similarity to small cell lung cancer (SCLC), another neuroendocrine tumor, chemotherapy regimens 
used to treat MCC were modeled after regimens used in SCLC [12]. Early cases were treated with drugs such as cyclophosphamide, doxorubicin and vincristine, but reports described limited efficacy [13]. More recently, platinum agents in combination with etoposide became the preferred chemotherapy regimen. Although MCC is often chemosensitive initially, responses are generally not durable. For example, one retrospective study reported an overall response rate of $55 \%$, but a median progression free survival of only 94 days [14]. Thus, there is a great need for discovering and testing new therapeutic options.

\section{The emergence of immune checkpoint inhibition}

Immune checkpoints are a cadre of molecules regulating $\mathrm{T}$ cell activation and proliferation which may become dysregulated or co-opted and allow the tumor to escape immune surveillance [15]. Discoveries in the 1980s and 1990s brought a greater understanding of the molecular underpinnings of self-tolerance and the ways in which immune checkpoint molecules control immunoregulatory signaling and $\mathrm{T}$ cell responses [16]. These discoveries led to the development of clinical agents targeting immune checkpoint ligands and receptors. The first of such inhibitors targeted CD152 (cytotoxic T-lymphocyte-associated antigen 4; CTLA-4). One such drug, ipilimumab, was the first in its class to demonstrate an improvement in overall survival in a clinical trial for patients with metastatic melanoma, which led to its approval by the FDA in 2011 [17]. Since then, other drugs have entered the market that target programmed cell death protein 1 (PD-1), another immune checkpoint receptor, or one of its associated ligands (PD-L1), and are now FDA-approved for a variety of cancers [18]. The success of immune checkpoint inhibitors in several cancer types and the immune susceptibility of MCC has renewed hope for developing more effective treatment options for patients with MCC.

\section{The immune system and Merkel cell carcinoma}

It has been long suspected that immune dysregulation plays a role in the development of MCC. Clinically, it was observed that chronically immunosuppressed patients, such as organ transplant recipients or those with HIV or B-cell malignancies, were at increased risk for developing MCC [19-22]. Early histological reports of primary MCC tumors demonstrated lymphocytic infiltration, evidence of MCC's immunogenic biology [23, 24]. More recently, tumor-infiltrating lymphocytes were found to correlate with a better prognosis, a finding which has been confirmed by genomic analysis of primary MCC tumors $[25,26]$. Of note, patients with an unknown primary lesion (e.g., those who present with a nodal metastasis only) have a better prognosis than those with a known primary lesion, suggesting that an immune-based response at the primary site leads to improved immunological tumor control overall [27-29].

In 2008, Feng and colleagues described an oncogenic Merkel cell polyomavirus (MCPyV), present in about $80 \%$ of MCC tumors. MCPyV creates a large T antigen that inactivates tumor suppressors p53 and RB. This discovery not only identified a causative factor for MCC, but also suggested a role for immune evasion in MCC's oncogenesis [30,31]. Viral antigens are expressed in MCC tumor cells and there is strong evidence for their recognition by innate and adaptive (i.e., cellular and humoral) immune elements [32]. Virus-negative MCCs may also be immunogenic, perhaps based on their high tumor mutation burden and neoantigens created as a result of ultraviolet light exposure [33]. However, despite their inherent immunogenicity, MCC tumors are able to evade the immune system through multiple mechanisms including the expression of immune checkpoint molecules. Notably, over $50 \%$ of Merkel-cell carcinomas express PD-1 on tumor-infiltrating lymphocytes and express PD-L1 on tumor cells [34]. The totality of these data provided a strong rationale for testing immune checkpoint blockers in patients with advanced MCC.

\section{Immune checkpoint inhibition in MCC}

Pembrolizumab was the first immune checkpoint inhibitor to demonstrate objective tumor regressions in patients with MCC [35]. Pembrolizumab is a humanized monoclonal antibody against PD-1 and is now FDA-approved for use in patients with a variety of cancers. In a phase 2, single-arm, multicenter study (ClinicalTrials.gov number NCT02267603), patients with advanced MCC who had not previously received systemic therapy were treated with pembrolizumab $2 \mathrm{mg} / \mathrm{kg}$ every three weeks for a maximum of two years or until disease progression, doselimiting toxicity, or complete response. Out of 26 patients, 4 experienced a complete response (CR) and 10 had a partial response (PR), for an ORR of 56\%. At 6 months, the progression-free survival rate was $67 \%$ and the duration of response ranged from 2.2 months to at least 9.7 months. $86 \%$ of responses were ongoing at last follow-up. (Table 1) These results prompted the addition of pembrolizumab for the treatment of disseminated MCC to the National Comprehensive Cancer Network (NCCN) guidelines [36]. Interestingly, objective regression was observed in both virus-positive and virus-negative tumors. PD-L1 expression did not seem to correlate with a higher likelihood of response to treatment as it has in other tumors [37].

In March 2017, a PD-L1 monoclonal antibody, avelumab, became the first FDA-approved treatment for MCC [38]. Approval was based on data from an open-label, single-arm, multi-center phase 2 clinical trial (JAVELIN Merkel 200; NCT02155647) [39]. In this study, 88 patients with advanced MCC who had progressed after receiving 
Table 1 Activity of PD-1-pathway-targeted agents in patients with advanced Merkel cell carcinoma

\begin{tabular}{|c|c|c|c|c|c|}
\hline Drug name & Drug class & $\mathrm{N}$ & Number of prior systemic therapies & ORR (\%) & Reference \\
\hline \multirow[t]{2}{*}{ Avelumab } & Anti-PD-L1 & 88 & $1-4$ & 33 & {$[40]$} \\
\hline & & 29 & 0 & 65 & {$[53]$} \\
\hline \multirow[t]{2}{*}{ Nivolumab } & Anti-PD-1 & 15 & 0 & $73^{*}$ & {$[44]$} \\
\hline & & 10 & $1-2$ & $50^{*}$ & \\
\hline Pembrolizumab & Anti-PD-1 & 26 & 0 & 56 & {$[35]$} \\
\hline
\end{tabular}

*RECIST v1.1, investigator assessed

chemotherapy received avelumab $10 \mathrm{mg} / \mathrm{kg}$ every 2 weeks. Updated outcomes at a median follow-up duration of 16.4 months revealed an ORR of $33 \%$, including $10 \mathrm{CRs}$ and 19 PRs [40]. (Table 1) Similar to the pembrolizumab trial, objective responses were observed regardless of PDL1 expression or MCPyV status. Responses were ongoing in $21 / 29$ patients $(72.4 \%)$ at last report. A separate, parallel cohort has been actively recruiting chemotherapy-naïve patients with advanced MCC. Preliminary data among 25 patients with $>6$ weeks of follow-up demonstrated an unconfirmed ORR of 64\% [41]. When compared to historical trials of patients with advanced MCC receiving chemotherapy, the durability of responses to avelumab appears substantially superior [14, 42, 43]. (Table 2 ).

Nivolumab is another monoclonal PD-1 antibody with clinical activity in advanced MCC. As part of the phase 1/ 2 multiple cohort CheckMate 358 study (NCT02488759), 25 patients with both treatment-naïve and previouslytreated, MCPyV-positive or -negative, advanced MCC were enrolled and treated with nivolumab $240 \mathrm{mg}$ every 2 weeks [44]. Among 25 patients, with a median follow-up of 51 weeks (range: 5-63 weeks), investigators observed a 64\% ORR. Arithmetically, the ORR was higher among the 15 treatment-naïve patients (73\%) compared with the 10 previously-treated patients (50\%), though these numbers are too small to reach statistical significance. The median duration of response was not reached. Consistent with findings in the two trials described above, objective responses occurred independent of PD-L1 expression and MCPyV status. Expansion cohorts on this trial are investigating the activity and safety of nivolumab in combination with ipilimumab or relatlimab (BMS986016, anti-LAG-3) in patients with advanced MCC or other virus-associated cancers. Of note, ipilimumab monotherapy has demonstrated durable anti-tumor activity in small case series of 5 chemotherapy-naïve patients with metastatic MCC [45].

\section{Adverse reactions}

The safety profiles of the PD-1/PD-L1 antibodies administered to patients with MCC appear similar to those from previous trials involving patients with other tumor types. Immune-mediated adverse reactions observed on the trials described above included adrenal insufficiency, colitis, hepatitis, myocarditis, nephritis, pneumonitis, thyroiditis, and transaminitis, among others. Of note, infusion-related reactions were observed with administration of avelumab, so premedication with an antihistamine and acetaminophen prior to the first four infusions of avelumab is now recommended [46]. In the avelumab trial, there were 5 grade 3 treatment-related adverse events reported in 4 (5\%) patients, including two cases of lymphopenia, and one case each of isolated elevations in serum creatine phosphokinase, alanine and aspartate aminotransferase (AST/ALT), or cholesterol. There were no treatmentrelated grade 4 adverse events or deaths observed in the trial [37]. Of patients receiving pembrolizumab, grade 3 or 4 treatment-related adverse events were observed in 15\% of patients [35]. Grade 4 events included myocarditis and elevated AST/ALT. Similarly, in CheckMate 358, grade 3 or 4 treatment-related adverse events were reported in $20 \%$ of patients and $12 \%$ had adverse events that led to nivolumab discontinuation [44].

\section{Conclusions and future directions}

Immunomodulatory therapies have had a profound impact on the cancer treatment landscape, and MCC is no exception. Indeed, response rates to single-agent

Table 2 Progression-free survival (PFS) outcomes for previously-treated patients with advanced MCC after treatment with avelumab (anti-PD-L1) compared with PFS rates from previous chemotherapy trials (historical controls)

\begin{tabular}{llll}
\hline Anti-neoplastic agent(s) & Median PFS, months $(95 \% \mathrm{Cl})$ & PFS rate at 12 months, \% $(95 \% \mathrm{Cl})$ & Reference \\
\hline Avelumab $(N=88)$ & $2.7(1.4-6.9)$ & $30(21-41)$ & {$[39]$} \\
Cowey $2017(n=20)$ & $2.1(1.0-3.2)$ & 0 & {$[42]$} \\
Becker $2016(n=34)$ & $3.0(2.6-3.1)$ & 0 & {$[43]$} \\
lyer $2016(n=30)$ & $2.0($ NA [range: $0.4-11.6])$ & $0^{*}$ & {$[14]$}
\end{tabular}

The most common second-line chemotherapy was topotecan. NA, not available; *based on PFS range (11-354 days) and Kaplan-Meier PFS estimates 
immune checkpoint inhibition seem to compare favorably to those of other tumor types [47]. With the recent FDA approval of avelumab for previously-treated advanced MCC, patients with MCC now have a new treatment option beyond chemotherapy. The results of the trials described above led to the inclusion of avelumab, pembrolizumab and nivolumab in the January 2018 NCCN guidelines as preferred treatment options for patients with disseminated disease [36]. Although data are still preliminary, it appears that rates of MCC regression in treatment-naïve patients treated with PD-1/PD-L1pathway blockers may exceed those of patients who were previously treated. These findings require validation in larger patient cohorts, but suggest that immune checkpoint blockade may be most efficacious when used in the first-line setting. Furthermore, responses appear to be durable, unlike those seen with cytotoxic chemotherapy and hence, these agents are becoming the new standard-of-care for treating patients with metastatic or unresectable MCC.

The immunogenic characteristics demonstrated by both MCPyV-positive and -negative Merkel cell tumors perhaps underlie its sensitivity to immuno-oncology agents. Ongoing and future trials aim to capitalize on this phenotype by interrogating and manipulating the tumor microenvironment and host immune system in order to develop more effective combinatorial regimens. One such trial combines localized radiotherapy or recombinant interferon beta and avelumab with or without cellular adoptive immunotherapy for patients with metastatic MCC. Both radiation and interferon beta can enhance the host immune response by upregulating MHC class I molecules. Combining either approach with polyclonal CD8+ T cells and a PD-L1 blocker (avelumab) may enrich the immune microenvironment by expanding molecular immune targets, allowing for anti-tumor $\mathrm{T}$ cell activation (NCT02584829). Another phase 2 study (NCT02465957) seeks to combine activated NK-92 natural killer cell infusions with ALT-803 (interleukin-15) in patients with advanced MCC. One emerging area of investigation is focused on determining when administration of immune checkpoint inhibition is most effective. For example, several clinical trials are investigating the utility of immune checkpoint blockers in the adjuvant setting (e.g., NCT02196961, NCT03271372) administered with or without radiotherapy. Combining these modalities may provide synergistic anti-tumor activity for patients with stage III MCC (i.e., regional nodal metastases), for whom adjuvant RT alone has, historically, not led to improvements in overall survival [48]. This approach follows successful adjuvant trials in other locoregionally advanced cutaneous malignancies (e.g., stage III melanoma) [49]. The neoadjuvant setting also provides an opportunity for administration of immune checkpoint inhibition. Early data from clinical trials suggest benefit, including in patients with melanoma [50]. Other therapies on the horizon include trials combining intralesional T-VEC (talimogene laherparepvec), an oncolytic, recombinant herpes simplex type-1 virus-based agent, with radiotherapy (NCT02819843) or nivolumab (NCT02978625). The FDA's recent approval of tisagenlecleucel [51], a chimeric antigen receptor $\mathrm{T}$ cell (CAR-T) therapy, for patients with $\mathrm{B}$-cell precursor acute lymphoblastic leukemia underscores the potential for this immune-based anti-cancer strategy. Given the role of MCPyV in driving MCC carcinogenesis, a future treatment approach may involve administration of genetically-modified CAR-T cells against MCPyV antigens.

Because MCC is a rare cancer, our understanding of the disease biology and the utility of novel therapies seems best strengthened by conducting international, multi-center, and cooperative group trials using novel study designs [52]. In the last few years, advances in our understanding of how immunotherapies can treat patients with MCC have brought hope and optimism to cancer researchers, clinicians and patients alike, and have laid a foundation for the continued development of safe and effective treatment regimens for patients with this rare, deadly disease.

\section{Abbreviations \\ ALT: alanine aminotransferase; AST: aspartate aminotransferase; CAR- T: chimeric antigen receptor T-cell; CTLA4: Cytotoxic T-lymphocyte-associated protein 4; DoR: Duration of response; MCC: Merkel cell carcinoma; MCPyV: Merkel cell polyomavirus; NCCN: National Comprehensive Cancer Network; ORR: Objective response rate; PD1: Programmed cell death protein 1; PDL1: Programmed cell death-ligand 1; PFS: Progression-free survival}

Availability of data and materials

Not Applicable.

Authors' contributions

ISC and EJL drafted the manuscript. All authors read, critically revised and approved the final manuscript.

Ethics approval and consent to participate

Not applicable.

\section{Consent for publication}

Not applicable.

\section{Competing interests}

ISC has no competing interests to declare.

SB has received advisory board honoraria from Genentech and EMD-Serono; his institution (University of Washington) has received research funding from EMD-Serono, Merck, BMS, Oncosec, and Immune Design.

HLK has served on advisory boards for Amgen, Celldex, Compass

Therapeutics, EMD Serono, Merck, Prometheus and Turnstone Biologics. He receives research funding from Amgen, EMD Serono, Merck, Prometheus and Viralytics. He serves on a speaker's bureau for Merck but all honoraria are returned to Rutgers University.

EJL is a consultant for Array BioPharma and Bristol-Myers Squibb. He receives institutional research funding from Bristol-Myers Squibb and Merck.

\section{Publisher's Note}

Springer Nature remains neutral with regard to jurisdictional claims in published maps and institutional affiliations. 


\section{Author details}

'Department of Oncology, Johns Hopkins University School of Medicine, Sidney Kimmel Comprehensive Cancer Center, and Bloomberg-Kimmel Institute for Cancer Immunotherapy, Baltimore, MD, USA. ${ }^{2}$ Department of Medicine/Medical Oncology, University of Washington and Fred Hutchinson Cancer Research Center, Seattle, Washington, USA. ${ }^{3}$ Department of Surgery, Massachusetts General Hospital, Boston, MA, USA. ${ }^{4}$ Melanoma and Cancer Immunology Programs, Johns Hopkins University School of Medicine, 1550 Orleans Street, Room 507, Baltimore, MD 21287, USA.

Received: 24 October 2017 Accepted: 7 March 2018

Published online: 23 March 2018

\section{References}

1. Albores-Saavedra J, Batich K, Chable-Montero F, Sagy N, Schwartz AM, Henson DE. Merkel cell carcinoma demographics, morphology, and survival based on 3870 cases: a population based study. J Cutan Pathol. 2010;37:20-7.

2. Heath $M$, Jaimes $N$, Lemos B, Mostaghimi A, Wang LC, Penas PF, Nghiem P. Clinical characteristics of Merkel cell carcinoma at diagnosis in 195 patients: the AEIOU features. J Am Acad Dermatol. 2008:58:375-81.

3. Hodgson NC. Merkel cell carcinoma: changing incidence trends. J Surg Oncol. 2005:89:1-4.

4. Fitzgerald TL, Dennis S, Kachare SD, Vohra NA, Wong JH, Zervos EE. Dramatic increase in the incidence and mortality from Merkel cell carcinoma in the United States. Am Surg. 2015;81:802-6.

5. Becker JC: Merkel cell carcinoma. Ann Oncol 2010, 21 Suppl 7:vii81-85.

6. Harms KL, Healy MA, Nghiem P, Sober AJ, Johnson TM, Bichakjian CK, Wong SL. Analysis of prognostic factors from 9387 Merkel cell carcinoma cases forms the basis for the new 8th edition AJCC staging system. Ann Surg Oncol. 2016;23:3564-71.

7. Schadendorf D, Lebbe C, Zur Hausen A, Avril MF, Hariharan S, Bharmal M, Becker JC. Merkel cell carcinoma: epidemiology, prognosis, therapy and unmet medical needs. Eur J Cancer. 2017;71:53-69.

8. Houben R, Schrama D, Becker JC. Molecular pathogenesis of Merkel cell carcinoma. Exp Dermatol. 2009;18:193-8.

9. Hasan S, Liu L, Triplet J, Li Z, Mansur D. The role of postoperative radiation and chemoradiation in merkel cell carcinoma: a systematic review of the literature. Front Oncol. 2013;3:276.

10. Allen PJ, Bowne WB, Jaques DP, Brennan MF, Busam K, Coit DG. Merkel cell carcinoma: prognosis and treatment of patients from a single institution. J Clin Oncol. 2005;23:2300-9.

11. Garneski KM, Nghiem P. Merkel cell carcinoma adjuvant therapy: current data support radiation but not chemotherapy. J Am Acad Dermatol. 2007; 57:166-9.

12. Feun LG, Savaraj N, Legha SS, Silva EG, Benjamin RS, Burgess MA. Chemotherapy for metastatic Merkel cell carcinoma. Review of the M.D. Anderson Hospital's experience. Cancer. 1988;62:683-5.

13. Pollack SV, Goslen JB. Small-cell neuroepithelial tumor of skin: a Merkel-cell neoplasm. J Dermatol Surg Oncol. 1982;8:116-22.

14. Iyer JG, Blom A, Doumani R, Lewis C, Tarabadkar ES, Anderson A, Ma C, Bestick A, Parvathaneni U, Bhatia S, Nghiem P. Response rates and durability of chemotherapy among 62 patients with metastatic Merkel cell carcinoma. Cancer Med. 2016:5:2294-301.

15. Pardoll DM. The blockade of immune checkpoints in cancer immunotherapy. Nat Rev Cancer. 2012;12:252-64.

16. Leach DR, Krummel MF, Allison JP. Enhancement of antitumor immunity by CTLA-4 blockade. Science. 1996;271:1734-6.

17. Hodi FS, O'Day SJ, McDermott DF, Weber RW, Sosman JA, Haanen JB, Gonzalez R, Robert C, Schadendorf D, Hassel JC, et al. Improved survival with ipilimumab in patients with metastatic melanoma. N Engl J Med. 2010;363:711-23.

18. Postow MA, Callahan MK, Wolchok JD. Immune checkpoint blockade in Cancer therapy. J Clin Oncol. 2015;33:1974-82.

19. Formica M, Basolo B, Funaro L, Mazzucco G, Segoloni GP, Piccoli G. Merkel cell carcinoma in renal transplant recipient. Nephron. 1994;68:399.

20. Penn I, First MR. Merkel's cell carcinoma in organ recipients: report of 41 cases. Transplantation. 1999:68:1717-21.

21. Engels EA, Frisch M, Goedert JJ, Biggar RJ, Miller RW. Merkel cell carcinoma and HIV infection. Lancet. 2002:359:497-8.

22. Howard RA, Dores GM, Curtis RE, Anderson WF, Travis LB. Merkel cell carcinoma and multiple primary cancers. Cancer Epidemiol Biomark Prev. 2006;15:1545-9.
23. Sibley RK, Dehner LP, Rosai J. Primary neuroendocrine (Merkel cell?) carcinoma of the skin. I. A clinicopathologic and ultrastructural study of 43 cases. Am J Surg Pathol. 1985;9:95-108.

24. Silva EG, Mackay B, Goepfert H, Burgess MA, Fields RS. Endocrine carcinoma of the skin (Merkel cell carcinoma). Pathol Annu. 1984;19(Pt 2):1-30.

25. Mott RT, Smoller BR, Morgan MB. Merkel cell carcinoma: a clinicopathologic study with prognostic implications. J Cutan Pathol. 2004;31:217-23.

26. Paulson $\mathrm{KG}$, lyer JG, Tegeder AR, Thibodeau $\mathrm{R}$, Schelter J, Koba S, Schrama D, Simonson WT, Lemos BD, Byrd DR, et al. Transcriptomewide studies of merkel cell carcinoma and validation of intratumoral CD8+ lymphocyte invasion as an independent predictor of survival. J Clin Oncol. 2011;29:1539-46.

27. Chen KT, Papavasiliou P, Edwards K, Zhu F, Perlis C, Wu H, Turaka A, Berger A, Farma JM. A better prognosis for Merkel cell carcinoma of unknown primary origin. Am J Surg. 2013;206:752-7.

28. Tarantola TI, Vallow LA, Halyard MY, Weenig RH, Warschaw KE, Weaver AL, Roenigk RK, Brewer JD, Otley CC. Unknown primary Merkel cell carcinoma: 23 new cases and a review. J Am Acad Dermatol. 2013;68:433-40

29. Fields RC, Busam KJ, Chou JF, Panageas KS, Pulitzer MP, Allen PJ, Kraus DH, Brady MS, Coit DG. Five hundred patients with Merkel cell carcinoma evaluated at a single institution. Ann Surg. 2011:254:465-73. discussion 473-465

30. Feng $H$, Shuda M, Chang Y, Moore PS. Clonal integration of a polyomavirus in human Merkel cell carcinoma. Science. 2008;319:1096-100.

31. Bhatia S, Afanasiev O, Nghiem P. Immunobiology of Merkel cell carcinoma: implications for immunotherapy of a polyomavirus-associated cancer. Curr Oncol Rep. 2011;13:488-97.

32. Schadendorf D, Nghiem P, Bhatia S, Hauschild A, Saiag P, Mahnke L, Hariharan S, Kaufman HL. Immune evasion mechanisms and immune checkpoint inhibition in advanced merkel cell carcinoma. Oncolmmunology. 2017:e1338237.

33. Goh G, Walradt T, Markarov V, Blom A, Riaz N, Doumani R, Stafstrom K, Moshiri A, Yelistratova L, Levinsohn J, et al. Mutational landscape of MCPyVpositive and MCPyV-negative Merkel cell carcinomas with implications for immunotherapy. Oncotarget. 2016;7:3403-15.

34. Lipson EJ, Vincent JG, Loyo M, Kagohara LT, Luber BS, Wang H, Xu H, Nayar SK, Wang TS, Sidransky D, et al. PD-L1 expression in the Merkel cell carcinoma microenvironment: association with inflammation, Merkel cell polyomavirus and overall survival. Cancer Immunol Res. 2013;1:54-63.

35. Nghiem PT, Bhatia S, Lipson EJ, Kudchadkar RR, Miller NJ, Annamalai L, Berry S, Chartash EK, Daud A, Fling SP, et al. PD-1 blockade with Pembrolizumab in advanced Merkel-cell carcinoma. N Engl J Med. 2016;374:2542-52.

36. Merkel cell carcinoma guidelines [www.nccn.org/professionals/physician_ gls/PDF/mcc.pdf.]

37. Garon EB, Rizvi NA, Hui R, Leighl N, Balmanoukian AS, Eder JP, Patnaik A Aggarwal C, Gubens M, Horn L, et al. Pembrolizumab for the treatment of non-small-cell lung cancer. N Engl J Med. 2015;372:2018-28.

38. Chin K, Chand VK, Nuyten DS. Avelumab: clinical trial innovation and collaboration to advance anti-PD-L1 immunotherapy. Ann Oncol. 2017;

39. Kaufman HL, Russell J, Hamid O, Bhatia S, Terheyden P, D'Angelo SP, Shih KC, Lebbe C, Linette GP, Milella M, et al. Avelumab in patients with chemotherapy-refractory metastatic Merkel cell carcinoma: a multicentre, single-group, open-label, phase 2 trial. Lancet Oncol. 2016;17:1374-85.

40. Kaufman HL, Russell JS, Hamid O, Bhatia ST P., D'Angelo SP, Shih KC, Lebbé CM M., Brownell I, Lewis KD, Lorch JHH A., et al: Durable responses to avelumab (anti-PD-L1) in patients with Merkel cell carcinoma progressed after chemotherapy: 1-year efficacy update. In American Association for Cancer Research annual meeting; 2017.

41. D'Angelo SP, Russell J, Hassel JC, Lebbe C, Chmielowski B, Rabinowits G, Terheyden P, Brownell I, Zwiener I, Bajars M. First-line (1L) avelumab treatment in patients (pts) with metastatic Merkel cell carcinoma (mMCC): preliminary data from an ongoing study. Proc Am Soc Clin Oncol. 2017;

42. Cowey CL, Mahnke L, Espirito J, Fox P, Helwig C, Oksen D, Bharmal M. Realworld outcomes of patients with metastatic Merkel cell carcinoma treated with second-line or later chemotherapy in a community oncology setting in the United States. Value Health. 2017;19:A717

43. Becker J, Lorenz E, Haas G, Helwig C, Oksen D, Mahnke L, Bharmal M. Evaluation of real world treatment outcomes in patients with metastatic merkel cell carcinoma (MCC) following second line chemotherapy. Ann Oncol. 2016;27:-1154P. 1154P

44. Topalian SL, Bhatia S, Hollebecque A, Awada A, Boer JPD, Kudchadkar RR, Goncalves A, Delord J-P, Martens UM, Picazo JML, et al: Abstract CT074: 
non-comparative, open-label, multiple cohort, phase $1 / 2$ study to evaluate nivolumab (NIVO) in patients with virus-associated tumors (CheckMate 358): efficacy and safety in Merkel cell carcinoma (MCC). Cancer Res 2017, 77: СТ074-СT074.

45. Winkler JK, Dimitrakopoulou-Strauss A, Sachpekidis C, Enk A, Hassel JC. Ipilimumab has efficacy in metastatic Merkel cell carcinoma: a case series of five patients. J Eur Acad Dermatol Venereol. 2017;

46. BAVENCIO(R) [package insert]. EMD Serono. Inc. Rockland: MA; 2017. https:// dailymed.nlm.nih.gov/dailymed/getFile.cfm?setid=5cd725a1-2fa4-408aa651-57a7b84b2118\&type $=$ pdf\&name $=5 c d 725 a 1-2 f a 4-408 a-a 651-$ $57 a 7 b 84 b 2118$.

47. Sharma P, Allison JP. The future of immune checkpoint therapy. Science. 2015;348:56-61.

48. Bhatia S, Storer BE, Iyer JG, Moshiri A, Parvathaneni U, Byrd D, Sober AJ, Sondak VK, Gershenwald JE, Nghiem P. Adjuvant radiation therapy and chemotherapy in Merkel cell carcinoma: survival analyses of 6908 cases from the National Cancer Data Base. J Natl Cancer Inst. 2016;108

49. Weber J, Mandala M, Del Vecchio M, Gogas HJ, Arance AM, Cowey CL, Dalle S, Schenker M, Chiarion-Sileni V, Marquez-Rodas I, et al: Adjuvant Nivolumab versus Ipilimumab in resected stage III or IV melanoma. N Engl J Med 2017, 377:1824-1835.

50. Rozeman EA, Blank CU, Akkooi ACJV, Kvistborg P, Fanchi L, Thienen JW, Stegenga B, Lamon B, Haanen JBAG, Schumacher T: Neoadjuvant ipilimumab + nivolumab (IPI+NIVO) in palpable stage III melanoma: updated data from the OpACIN trial and first immunological analyses. J Clin Oncol 2017, 35:9586-9586.

51. Ledford H. Support for risky cancer therapy. NATURE PUBLISHING GROUP MACMILLAN BUILDING, 4 CRINAN ST, LONDON N1 9XW. In: ENGLAND: 2017.

52. Griggs RC, Batshaw M, Dunkle M, Gopal-Srivastava R, Kaye E, Krischer J, Nguyen T, Paulus K, Merkel PA. Rare diseases clinical research N: clinical research for rare disease: opportunities, challenges, and solutions. Mol Genet Metab. 2009;96:20-6.

53. D'Angelo SP, Russell J, Hassel J, Lebbe C, Chmielowski B, Rabinowits G, Terheyden P, Brownell I, Zwiener I, Bajars M, et al. Avelumab treatment in chemotherapy-nä"ive patients with distant metastatic Merkel cell carcinoma (mMCC). In: European Society for Medical Oncology 2017 congress scientific committee; September 8-12. Spain: Madrid; 2017. p. 2017.

\section{Submit your next manuscript to BioMed Central and we will help you at every step:}

- We accept pre-submission inquiries

- Our selector tool helps you to find the most relevant journal

- We provide round the clock customer support

- Convenient online submission

- Thorough peer review

- Inclusion in PubMed and all major indexing services

- Maximum visibility for your research

Submit your manuscript at www.biomedcentral.com/submit 